\title{
Perivascular iron deposition and other vascular damage in multiple sclerosis
}

\author{
C W M ADAMS \\ From the Division of Histopathology, United Medical and Dental Schools of Guy's and St Thomas's Hospitals, \\ University of London, $U K$
}

SUMMARY Evidence of damage to cerebral vein walls was sought in 70 cases of multiple sclerosis. Seventy control cases were also examined. The multiple sclerosis cases showed venous intramural fibrinoid deposition (7\%), recent haemorrhages (17\%), old haemorrhages revealed by haemosiderin deposition (30\%), thrombosis $(6 \%)$ and thickened veins $(19 \%)$. In all, $41 \%$ of all multiple sclerosis cases showed some evidence of vein damage. Occasional control cases showed haemosiderin deposition in the brain but, unlike the multiple sclerosis cases, these were diffuse and almost entirely related to coexistent cardiovascular or cerebrovascular disease. Haemosiderin deposition was common in the substantia nigra and other pigmented nuclei in all cases. It is concluded that the cerebral vein wall in multiple sclerosis is subject to chronic inflammatory damage, which promotes hae- $\vec{i}$ morrhage and increased permeability, and constitutes a form of vasculitis.

Involvement of the cerebral venous system in multiple sclerosis has been recognised for many years. In particular, plaques are centred on small veins or venules ${ }^{1}$ and extend outwards therefrom along branch veins as Dawson's fingers. ${ }^{23}$ The veins in acute cases are usually the site of a lymphocyte-plasma cell infiltrate, which varies in intensity between modest to moderately severe. Chronic cases show less perivenular infiltration, which is often absent in the inactive or "burnt-out" case. ${ }^{4-8}$ In some active acute cases of multiple sclerosis the vein walls themselves are heavily infiltrated with inflammatory cells, but without infiltration of the adventitia and perivascular tissues. Chronic plaques often contain vein walls thickened by collagen. ${ }^{9}$ Although these processes are nonspecific, they do indicate that the vein wall is implicated in the inflammatory process, and is thereby damaged and thickened, partly analogous to the thickening in endarteritis obliterans in arteries passing through foci of chronic inflammation. Nevertheless, it could be held that the vein wall is merely acting as a conduit for inflammatory cells but, if so, it

Address for reprint requests: Professor C W M Adams, Department of Pathology, Medical School, Guy's Hospital, St Thomas's Street, London SE1 9RT, UK.

Received 12 May 1987 and in revised form 19 August 1987. Accepted 29 August 1987 should return to normality after subsidence inflammation.

The purpose of this study was to determine to whad extent vein walls are damaged in multiple sclerosi and whether such inflammatory damage constitutes form of vasculitis.

\section{Methods}

Paraffin blocks were prepared or recut from brain samples from 70 cases of multiple sclerosis, 64 random control cases obtained at necropsy and six cases of cerebral infarction or haemorrhage. The stroke cases were taken as "positive controls" for the presence of haemosiderin derived from haemorrhage. The multiple sclerosis cases were obtained from the MRC multiple sclerosis tissue bank at Guy's Hospital and from the Department of Neuropathology, Runwell Hospital, Wickford, Essex. Blocks usually included central white matter, third, lateral and fourth ventricles, brainstem, pons, medulla and cerebellum. Approximately 350 areas of multiple sclerosis brain and 200 areas from the control brains were studied. Apart from routine stains, sections were stained with Perls' potassium ferrocyanide technique for haemosiderin (iron), a MSB-trichrome method, phosphotungstic acid-haematoxylin, luxol fast blue, solochrome or other myelin stain, Holzer's method and, sometimes, with anti-fibrin peroxidase.

\section{Results}

The results are summarised in the table, and represen260 
Table Vein wall damage and iron deposition in multiple sclerosis and control brains*

\begin{tabular}{|c|c|c|c|}
\hline \multicolumn{2}{|l|}{$\begin{array}{l}\text { Multiple sclerosis } \\
(70)\end{array}$} & \multirow{3}{*}{$\begin{array}{l}\begin{array}{l}\text { Controls } \\
(64)\end{array} \\
\mathrm{Nil} \\
\mathrm{Nil}\end{array}$} & \multirow{3}{*}{$\begin{array}{l}\begin{array}{l}\text { Stroke } \\
(6)\end{array} \\
2(100 \%)\end{array}$} \\
\hline Intramural fibrinoid & $5(7 \%)+$ & & \\
\hline $\begin{array}{l}\text { Recent haemorrhage } \\
\text { Old haemorrhage }\end{array}$ & $12(17 \%) \dagger$ & & \\
\hline (haemosiderin) & $21(30 \%)_{+}^{+}$ & $4(6 \cdot 3 \%) \S$ & $4(100 \%)$ \\
\hline Thrombosis & $4(6 \%) \dagger$ & Nil & \\
\hline Collagenised thick & & & \\
\hline veins & $13(19 \%) \dagger$ & Nil & \\
\hline Total cases implicated & $29(41 \%)$ & $10(12 \cdot 5 \%)$ & \\
\hline
\end{tabular}

*Excluding changes in pigmented muclei of brainstem; see text.

$\dagger$ No case associated with either cardiovascular or cerebrovascular disease.

${ }_{\ddagger}$ Four of 21 multiple sclerosis cases containing haemosiderin had coexistent cardiovascular disease. Haemosiderin in multiple sclerosis brains was plaque-related in 20 of 21 cases.

$\S$ Three of four control cases containing haemosiderin had coexistent cardiovascular disease.

tative findings are illustrated in figs 1 to 4 . Active plaques were identified by the presence of a perivenular lymphocytic infiltration, an often irregular or shelving edge to the lesion and increased cellularity of the plaque or its edge. Chronic or subactive plaques showed trivial or absent lymphocytic infiltration, an often sharp edge, a much reduced cellular population and frequent thick-walled vessels.

Five active cases of multiple sclerosis $(7 \%$ of the total) showed fibrin (fibrinoid) within the vein wall (fig 1), while recent haemorrhages (fig 2) were found in 12 cases $(17 \%)$. Two of these haemorrhages were older and brown pigment was seen within attendant macrophages. Haemosiderin, revealed with Perls' method, was seen in plaques or within $2 \mathrm{~mm}$ of the plaque edge in 21 cases $(30 \%)$. It sometimes lay in ill-defined pallisades at the plaque edge (fig 3), sometimes within microglia and, most commonly, lay within vein walls (figs $2-3$ ).

In active cases, haemosiderin was sometimes found in areas of intense oedema and myelin pallor, often associated with marked perivenular lymphocytic cuffing. Only one case of multiple sclerosis showed haemosiderin with no relation to plaques, edges of plaques or areas of oedema and myelin pallor: there was no generalised vascular disease in this case.

In four chronic cases of multiple sclerosis $(6 \%)$,

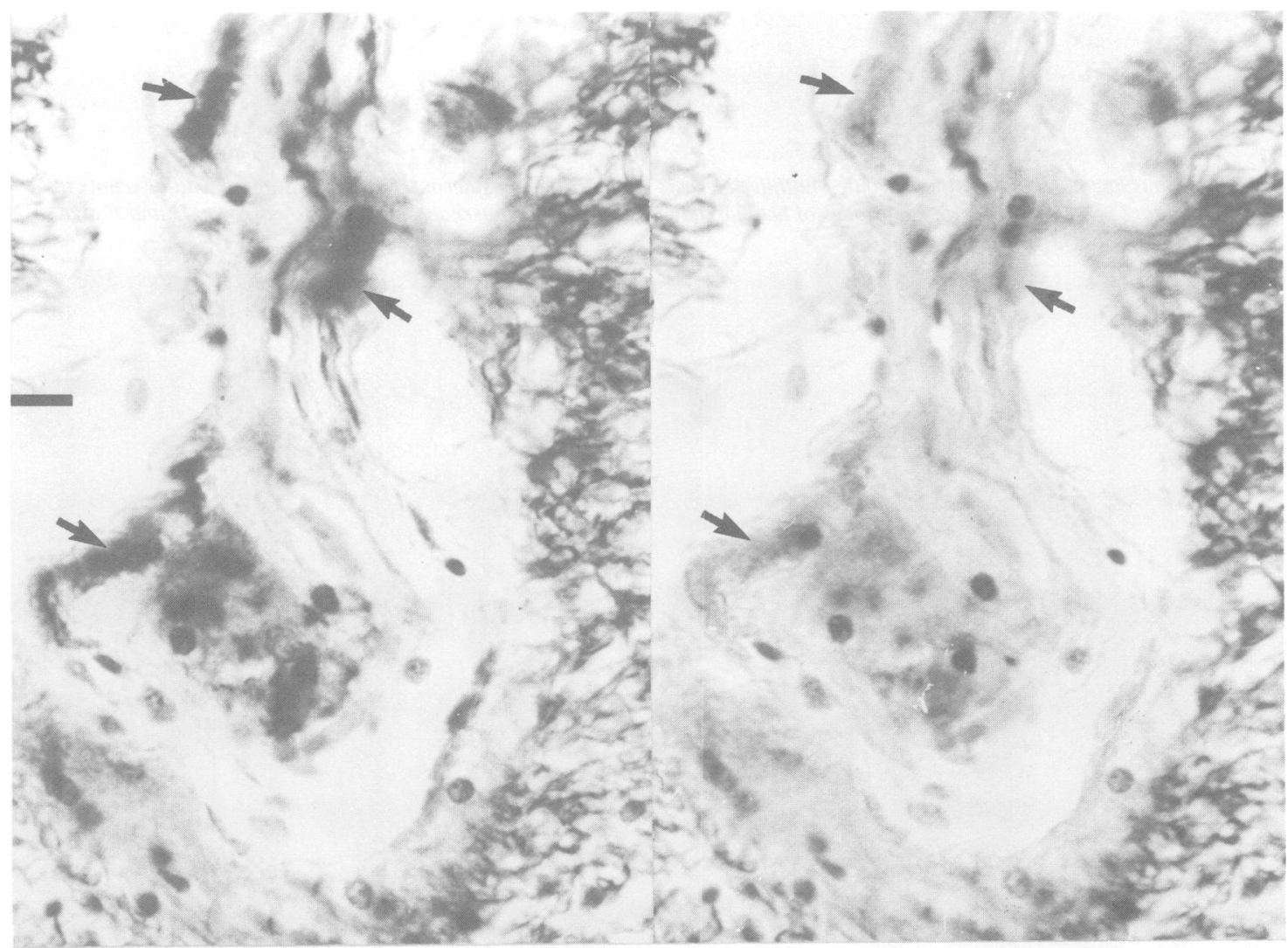

Fig 1 Venule at edge of plaque of active multiple sclerosis, showing fibrinoid (arrows) stained red in vein wall. Left: blue filter. Right: red filter. $M S B$-trichrome. Bar $=20 \mu \mathrm{m}$. 


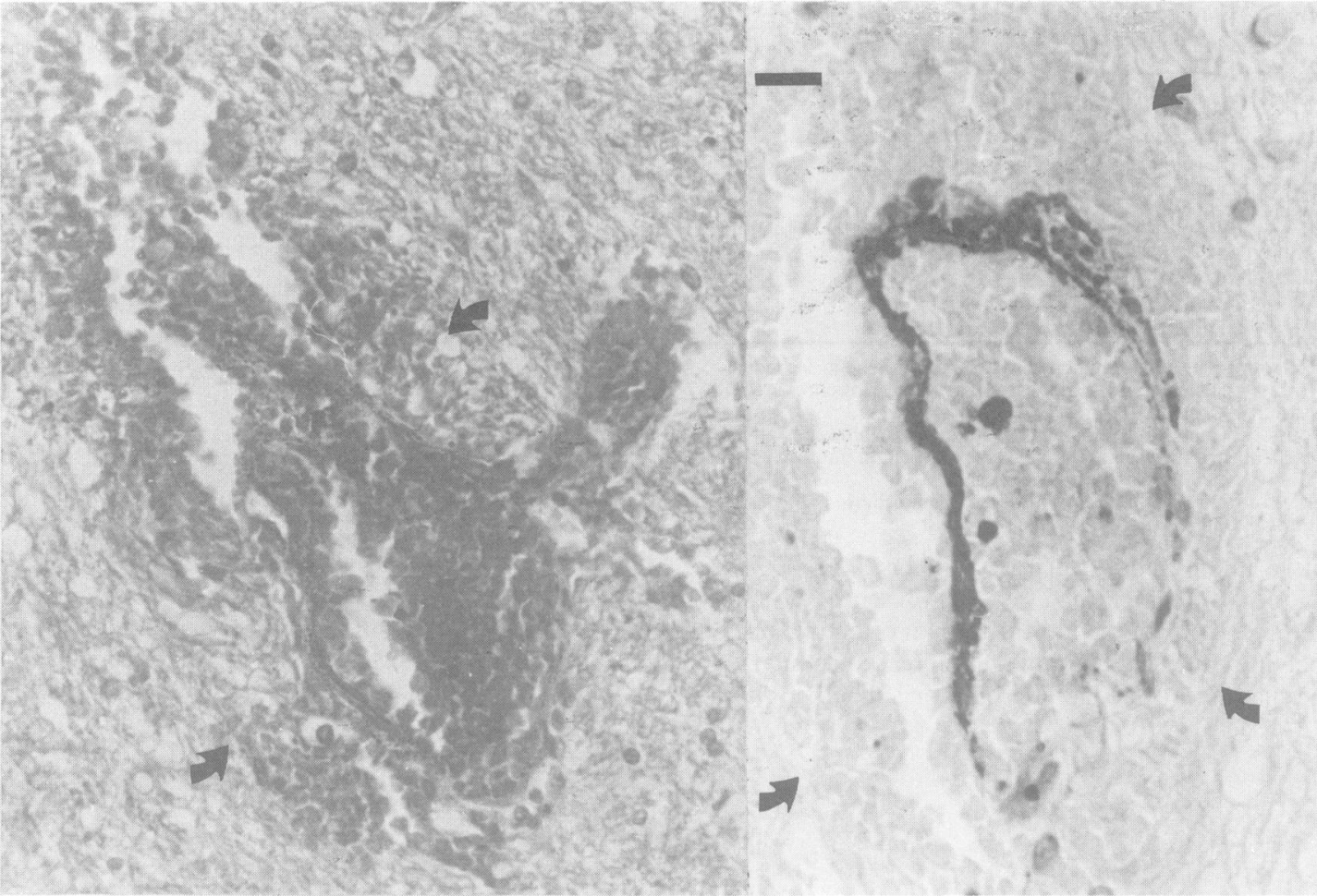

Fig 2 Venous haemorrhages within chronic multiple sclerosis plaques. Haemosiderin (iron) deposition in wall of venule at right; indicating present and previous episodes of haemorrhage. Limits of haemorrhages indicated by arrows. $H$ and $E$ and Perl's ferrocyanide. Bar $=20 \mu \mathrm{m}$.

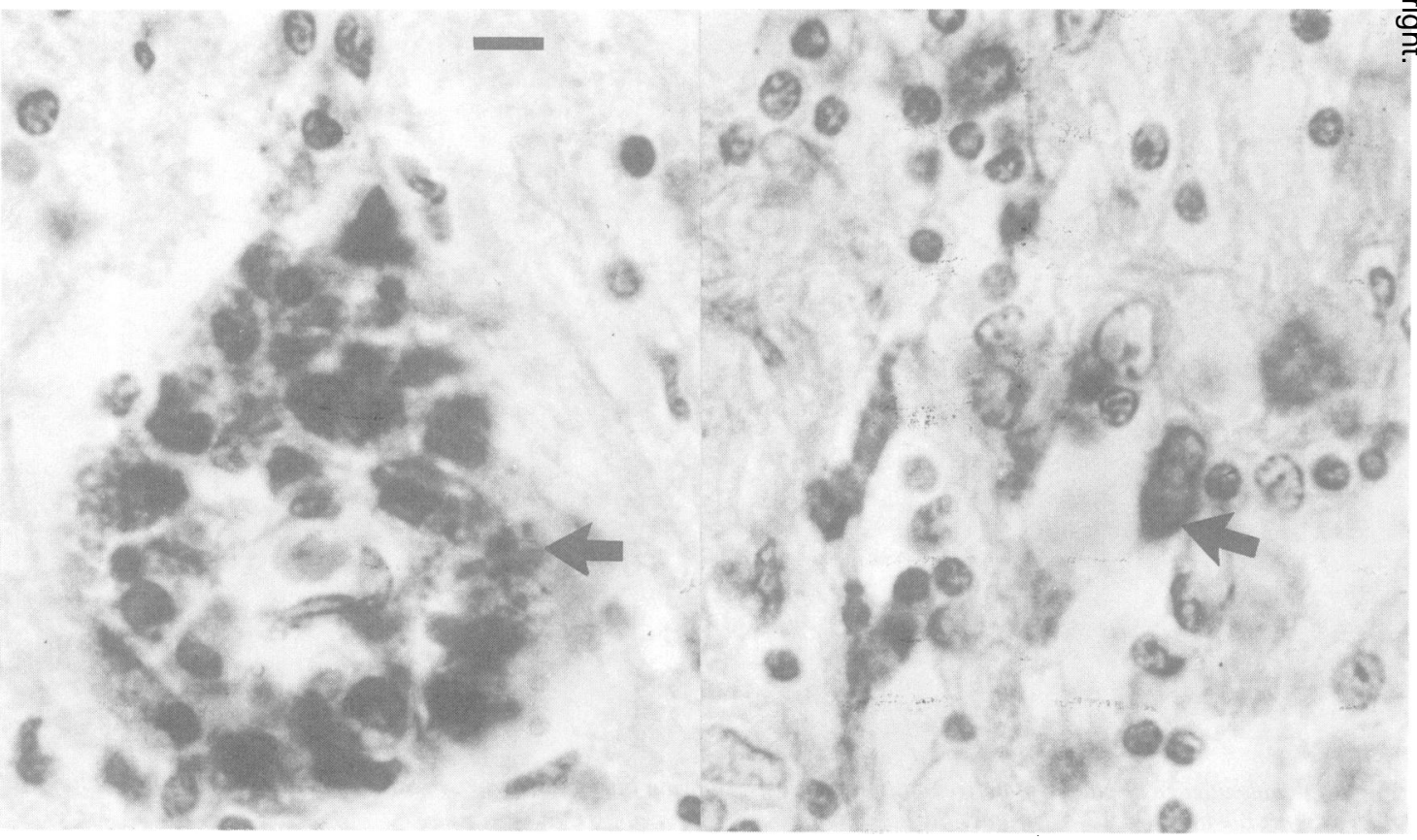

Fig 3 Haemosiderin (iron deposition) in wall of small venule in chronic multiple sclerosis plaque (left), and at edge of active multiple sclerosis plaque (right). Iron indicated by arrows. Perl's ferrocyanide, red filter. Bar $=12 \mu \mathrm{m}$. 
there were venous thrombi or encrustations presumed to have arisen by organisation of thrombi (fig 4). In one case thrombosis was accompanied by local haemorrhage, but neither this nor other thrombotic cases appeared to be early lesions. Chronic lesions in 13 cases $(19 \%)$ contained thick-walled veins, whose whole thickness was uniformly collagenised (fig 4).

The findings in the control and stroke cases are summarised in the table. The ten control and stroke cases that showed parenchymal or perivascular haemosiderin (apart from iron in the pigmented brainstem nuclei, see below), suffered from cerebrovascular disease (six), coronary heart disease and hypertension (two), congestive heart failure (one), and neoplasia (one). The cerebrovascular cases (infarction or haemorrhage) showed massive iron deposition around the lesions, and served as positive controls. One cerebrovascular case showed subpial haemosiderin deposition $^{1011}$ and this was presumed to result from previous subarachnoid haemorrhage. Some sections showed artefactual staining with ferrocyanide, either in the form of focal fine vesiculate staining or as coarse granules scattered over the surface of the section.

All but three of the 33 control cases, where there was good sampling of the pigmented brain stem nuclei, showed moderate amounts of haemosiderin deposition in the substantia nigra, olive or areas immediately adjacent to these nuclei.

No control case showed intramural deposition of fibrinoid. With the obvious exception of the six cerebrovascular accident cases, the control cases showed no recent haemorrhages or thromboses.

\section{Discussion}

The observations reported here indicate that veins in or around multiple sclerosis plaques suffer more damage than would be expected by simple involvement in an acute inflammatory episode. This damage is seen as occasional deposits of fibrin or fibrinoid within the vein wall ( $6 \%$ of cases), frequent recent haemorrhages $(17 \%)$, or residual haemosiderin as evidence of past haemorrhage $(30 \%)$ in direct relationship to plaques, and occasional thromboses within plaque veins $(6 \%)$. Such thromboses, however, do not appear to be associated with early plaques, in accord with Dow and Bergland $^{12}$ and with Zimmerman and Netsky. ${ }^{13}$

Capillary haemorrhages have been described in multiple sclerosis ${ }^{1415}$ and occasional venous hae-

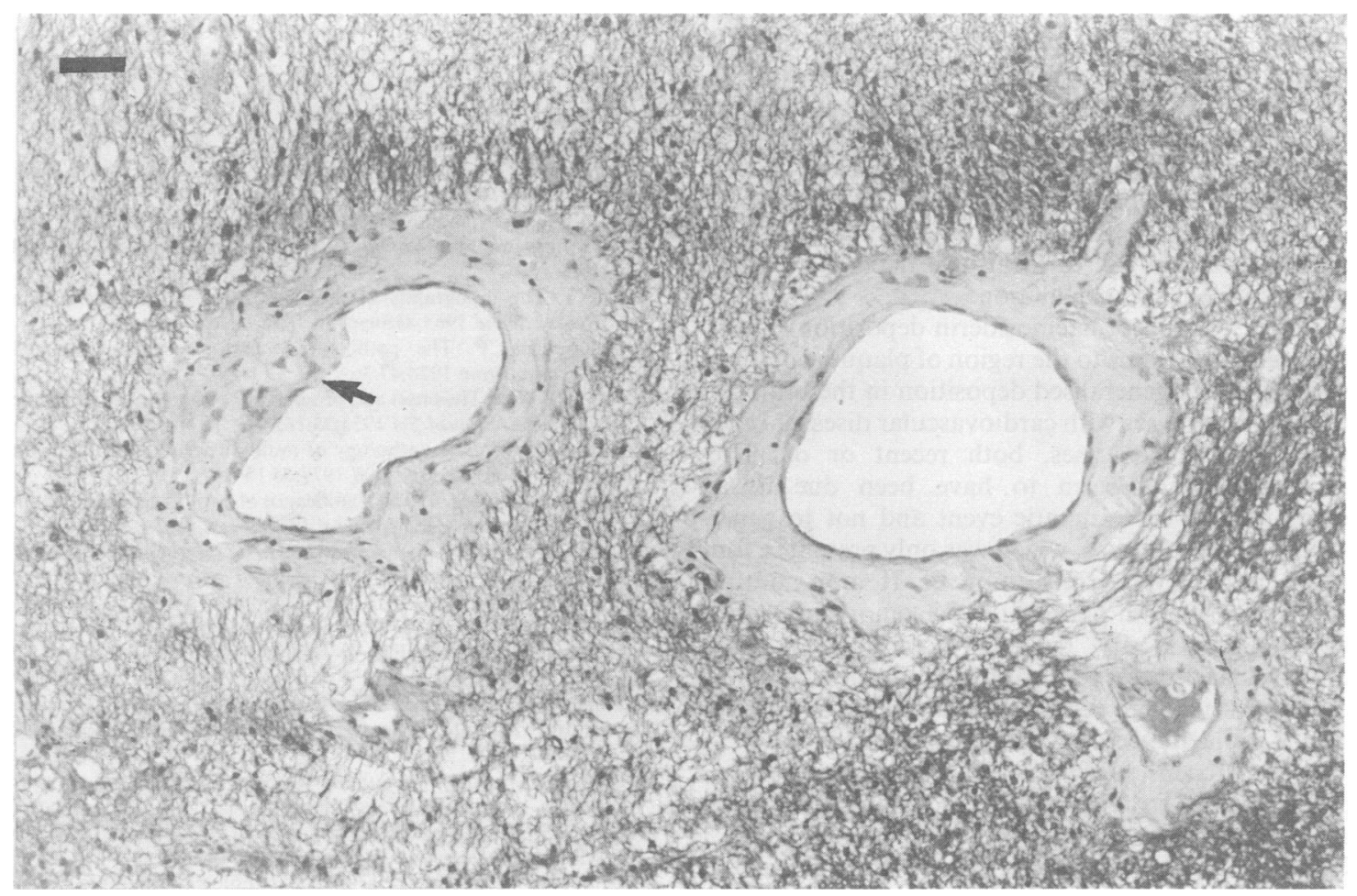

Fig 4 Thickened collagenised small veins in chronic periventricular plaques of multiple sclerosis. Arrow marks endothelial encrustation. Blue filter, $M S B$-trichrome. Bar $=50 \mu \mathrm{m}$. 
morrhages have also been previously recorded, ${ }^{10}$ but not as frequently as reported here. Venous haemorrhage in multiple sclerosis has also been compared, in terms : of pathogenesis, with the ring haemorrhages in another demyelinating disease, acute haemorrhagic leucoencephalomyelitis. ${ }^{17}$ Vehous haemorrhages in the brain could be held to be terminal anoxic or even traumatic events, but the location of haemorrhage and haemosiderin in the vicinity of plaques and the resulting local macrophage response is difficult to explain on the basis of a terminal or agonal event. Moreover, two substantial haemorrhages into multiple sclerosis plaques have been recorded clinically and, after successful neurosurgical evacuation of the clot, both patients made satisfactory recoveries. ${ }^{18}$

Walton and Kaufman ${ }^{19}$ examined 32 blocks from 13 subjects with multiple sclerosis but only detected haemosiderin in two of 13 multiple sclerosis subjects, apart from iron deposition in the substantia nigra in two further cases. Conversely, Craelius and colleagues $^{20}$ found haemosiderin deposits, similar to those reported in this paper, in all of the five multiple sclerosis cases examined.

Although haemosiderin deposition is encountered in occasional "normal" brains, it is usually of a more diffuse nature and is less frequently located in the walls of blood vessels. Iron deposition in the substantia nigra is a recognised finding in the normal brain. The incidence of haemosiderin deposition in the multiple sclerosis brain is $30 \%$, while, with the exception of the pigmented nuclei, the real incidence of such deposition in the brain of non-multiple sclerosis cases is $6 \%$. Cerebral infarction or haemorrhage was expected to give rise to local iron deposition and is excluded from this calculation.

The restriction of haemosiderin deposition in multiple sclerosis brains to the region of plaques contrasts with the more generalised deposition in the brains of those control cases with cardiovascular disease. In the multiple sclerosis cases, both recent or old haemorrhage would seem to have been due to an inflammatory or traumatic event and not to generalised vascular disease, which was only present in four of 33 cases. Conversely, nine of 10 non-multiple sclerosis cases with recent or old haemorrhage in the brain were suffering from cardiovascular or cerebrovascular disease. (Recent haemorrhage is assumed if extravascular red cells are found; old haemorrhage results in haemosiderin deposition.)

It has been suggested that multiple sclerosis patients suffer from splenic haemosiderosis, and that this is relevant to iron deposition in the brain. ${ }^{16}$ However; "Allen and colleagues ${ }^{21}$ were unable to confirm this in a survey of spleens from 67 necropsies on multiple sclerosis subjects.
The results reported here reinforce the view that damage to the vein wall is an important aspect of the pathology of the multiple sclerosis plaque. The vasculitis caused is different from and of a more modest nature than that, for example, in systemic lupus or polyarteritis nodosa but is, nevertheless, enough to cause haemorrhage, and structural and permeability changes in the vessel wall.

The term proposed by Lendrum ${ }^{22}$ for a wide range of vasculitic disorders is plasmatic vasculosis, and the damage to the vein wall in multiple sclerosis could be regarded as causing a minor degree of such plasmatic vasculosis.

Inflammatory and reparative changes in the vein wall might be exacerbated by pulsations or surges in intracranial venous pressure ${ }^{2324}$ and may result in increased permeability of the multiple sclerosis plaque, as shown at necropsy, ${ }^{25}$ by immunohistochemistry ${ }^{26}$ and by brain scan. ${ }^{27}$

The author is grateful to the Medical Research Council for a grant to aid this work and to the staff of the MRC Neuropathology Laboratory, Runwell Hospital, Wickford, Essex for help and providing some of the multiple sclerosis and control materialo in used. The technical help of Mr Stefan Buk is gratefully acknowledged.

\section{References}

1 Dawson JW. The histology of disseminated sclerosis. Trans $R$ So Edin 1916;108:397-9.

2 Fog T. Vessel-plaque relationships and CSF and brain tissue changes in multiple sclerosis. Acta Neurol Scand 1964;40Suppl 18:9-15.

3 Fog T. The topography of plaques in multiple sclerosis. Acta Neurol Scand 1965;41Suppl 15:1-162.

4 Symmonds CP. The pathological anatomy of disseminated sclerosis. Brain 1924;47:36-56.

5 Adams CWM. The onset and progression of the lesion in multiple sclerosis. J Neurol Sci 1975;25:165-82.

6 Adams CWM. The pathology of multiple sclerosis: progression of the lesion. Br Med Bull 1977;33:15-20.

7 Guseo A, Jellinger K. The significance of perivascular infiltration in multiple sclerosis. $J$ Neurol 1975;211:51-60.

8 Tanaka R, Iwasaki Y, Koprowski H. Ultrastructural studies of perivascular cuffing cells in multiple sclerosis brain. Am J Pathol 1975;81:467-78.

9 Adams CWM, Poston RN, Buk SJ, Sidhu YS, Vipond $\mathbf{H}$ Inflammatory vasculitis in multiple sclerosis. $J$ Neurol Sci 1985;69:269-83.

10 Tomlinson BE, Walton JN. Superficial haemosiderins of the central nervous system. $J$ Neurol Neurosurg Psychiatry 1964;27:332-9.

11 Braham J, Wolman M. Subpial siderosis of the central nervous system. Acta Neuropathol 1965;4:559-62.

12 Dow RS, Bergland G. Vascular pattern of lesions of multiple sclerosis. Arch Neurol Psychiatry 1942;47:1-18.

13 Zimmerman HM, Netsky MG. The pathology of multiple sclerosis. Res Pub Ass Rev Nerv Ment Dis 1950;28:271-99.

14 Siemerling E, Raecke E. Beitrag zur Klinik und Pathologie der 
Multiplen Sklerose mit besonderer Berucksikchtiging ihrer Pathogenese. Arch Nerve Psychiat Nervenkrank (Berlin) 1914;53:385-564.

15 Brown WJ. The capillaries in acute and subacute multiple sclerosis plaques. A morphometric analysis. Neurology 1978;28:84-92.

16 Lumsden CE. The neuropathology of multiple sclerosis. In: Vinken PJ, Bruyn GW, eds. Handbook of Clinical Neurology. Vol 19. Amsterdam: North Holland Publishers, 1970:217-309.

17 Chou SM. Acute haemorrhagic leucoencephalitis as vasculinomyelopathy. J Neuropathol Exp Neurol 1982;41:357.

18 Jankovic J, Derman H, Armstrong D. Haemorrhagic complications of multiple sclerosis. J Neurol Neurosurg Psychiatry 1980;43:76-81.

19 Walton JC, Kaufmann JCE. Iron deposits and multiple sclerosis. Arch Pathol 1984;108:755-6.

20 Craelius W, Migdal MW, Luessenhop CP, Sugar A, Mihalakis I. Iron deposits surrounding multiple sclerosis plaques. Arch Pathol 1982:106:397-9.
21 Allen IV, Millar JHD, Hutchinson MJ. General disease in 120 necropsy proven cases of multiple sclerosis. Neuropathol Appl Neurobiol 1978;4:279-84.

22 Lendrum AC. The hypertensive diabetic kidney as a model of the so-called collagen diseases. J Can Med Assoc 1963;88:442-52.

23 Schelling F. Damaging venous reflux into the skull or spine: relevance to multiple sclerosis. Medical Hypotheses 1986;21:141-8.

24 Portnoy HD, Chopp M, Branch C, Shannon M. CSF and venous pulse waves; a look at myogenic autoregulation. In: Auer LM, Heppner F, eds. The Cerebral Veins. Wien: Springer-Verlag, 1983:213-21.

25 Broman T. Blood brain damage in multiple sclerosis. Supravital test observations. Acta Neurol Scand 1964;Suppl 10:21-4.

26 Esiri MM. Multiple sclerosis: a quantitative and qualitative study of immunoglobulin containing cells in the central nervous system. Neuropathol Appl Neurobiol 1980;6:9-21.

27 Engel T, Havidberg A, Uhrenheldt A. Multiple sclerosisperiphlebitis retinae et cerebrospinalis. Acta Neurol Scand 1984;69:293-7. 